\title{
Measurement of Suitability of a Haptic Device in a Virtual Reality System
}

\author{
Jose San Martin $^{1}$ and Gracian Trivino ${ }^{2}$ \\ ${ }^{1}$ Universidad Rey Juan Carlos, 28933 Móstoles, Spain \\ jose.sanmartin@urjc.es \\ ${ }^{2}$ European Centre for Soft Computing, 33600 Mieres, Spain \\ gracian.trivino@softcomputing.es
}

\begin{abstract}
In the context of the optimization of the mechanical platform of a virtual reality system involving a haptic device, this paper introduces two tools in order to help the designer for obtaining the best positioning of the device respect to the application workspace. With this purpose we have defined a measure called Average Volumetric Manipulability, of how the application workspace fits in with the volume where haptic device provides its best performance. Also, we have defined other measure called Useful Manipulability which takes in account the frequency with which each zone of the application workspace is visited during the simulation process. The practical use of these measures is demonstrated using them during the design and development of a real application.
\end{abstract}

Keywords: Virtual reality, Haptic interface, Manipulability, Mechanical Performance.

\section{Introduction}

The design of a virtual environment in occasions involves the integration of different mechanical devices. The mechanical system can include manipulators that, depending on the configuration can reach more or less easily the different points of the workspace.

In case of not redundant manipulators, every point of the space can be only reached by a certain configuration of joints of the kinematics chain. The value of the angle of every joint, the geometry of arms of the manipulator, besides the relation that exists between the length of links of a manipulative arm, as main characteristics, determine the efficiency in the transmission of force and speed to the end of the manipulator (End Effector). A criterion of design of a system that contains haptic devices is maximizing this efficiency. A tool to obtain this goal will be to quantify the quality of the relative geometric positioning of each manipulator respect to the virtual environment workspace.

This work is part of a multidisciplinary project for the development of a Minimally Invasive Surgery Trainer (MIST) [1] where we have needed to optimize the mechanical relative positioning of the haptic devices in the system platform. 


\section{Manipulability}

Manipulability of a device is its ability to move freely in all directions into the workspace [2]. The first formulation that allowed a mathematical simple quantification was brought up by Yoshikawa [3]. We use the formulation of Manipulability proposed by Cavusoglu et al. [4]:

$$
\mu=\sigma_{\min }\left(J_{u}\right) / \sigma_{\max }\left(J_{u}\right) .
$$

Where:

$\sigma_{\min }$ and $\sigma_{\max }$ are the minimum and maximum singular values of $\mathbf{J}_{\mathrm{u}}$, upper half of the manipulator Jacobian matrix.

So the first step in the study of Manipulability is the analysis of the kinematics of a manipulator, in this case we have used the PHANToM OMNi of SensAble Technologies (Fig. 1).

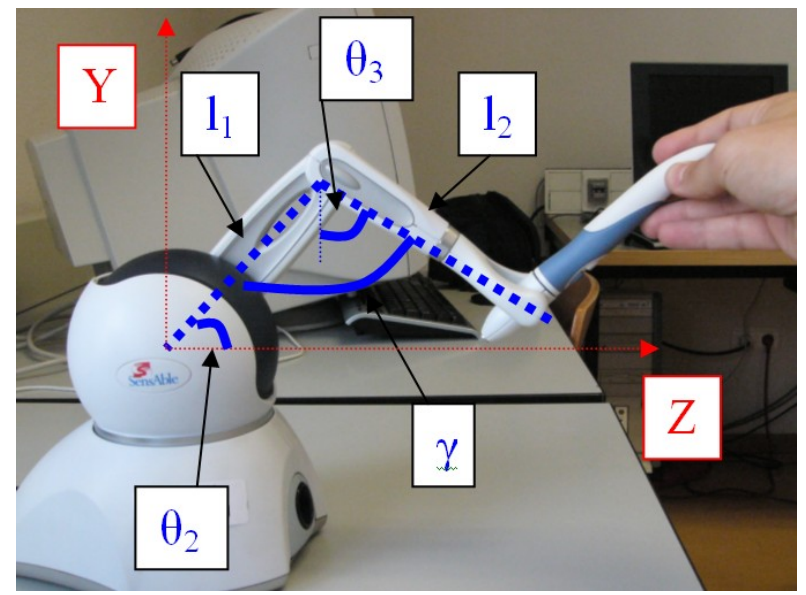

Fig. 1. OMNi arms $l_{1}=129 \mathrm{~mm}$ y $l_{2}=133 \mathrm{~mm}$. Angle inter-arms $\gamma$. Angles $\theta_{1}$ y $\theta_{2}$.

From the geometrical relations we obtain:

$$
\begin{aligned}
& \mathrm{x}=\left(\mathrm{l}_{1} \cos \theta_{2}+\mathrm{l}_{2} \sin \theta_{3}\right) \sin \theta_{1} \\
& \mathrm{y}=\mathrm{l}_{1} \sin \theta_{2}-\mathrm{l}_{2} \cos \theta_{3} \\
& \mathrm{z}=\left(\mathrm{l}_{1} \cos \theta_{2}+\mathrm{l}_{2} \sin \theta_{3}\right) \cos \theta_{1}
\end{aligned}
$$

The analysis of the kinematics of the device allows us to calculate the Jacobian matrix associated to this device [5]. 


$$
J=\left(\begin{array}{ccc}
l_{1} \cos \left(\theta_{2}\right)+l_{2} \sin \left(\theta_{3}\right) & 0 & 0 \\
0 & l_{1} \cos \left(\theta_{2}-\theta_{3}\right) & 0 \\
0 & -l_{1} \sin \left(\theta_{2}-\theta_{3}\right) & l_{2} \\
0 & 0 & -1 \\
\cos \left(\theta_{3}\right) & 0 & 0 \\
\sin \left(\theta_{3}\right) & 0 & 0
\end{array}\right)
$$

From Jacobian we calculate using (1) the Manipulability measure. Figure 2 shows the YZ-map of Manipulability obtained for the OMNi.

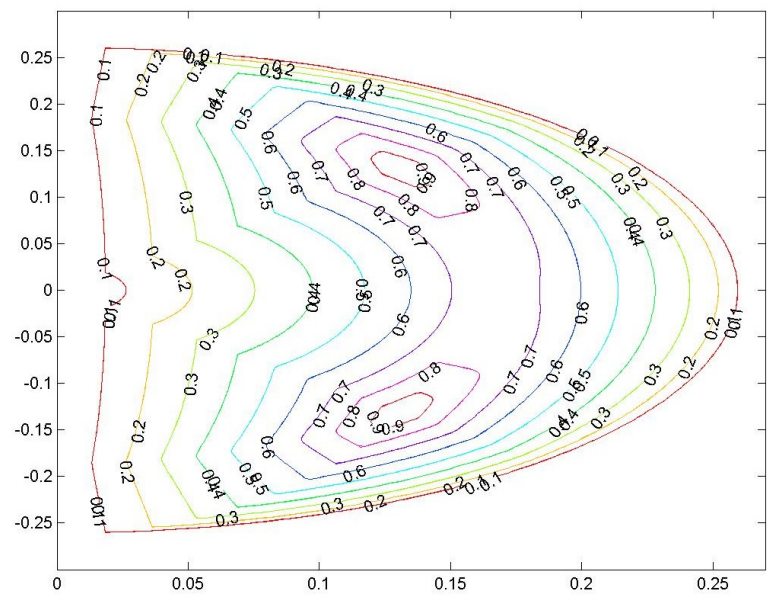

Fig. 2. Map of Manipulability values for plane YZ. Axes in millimetres.

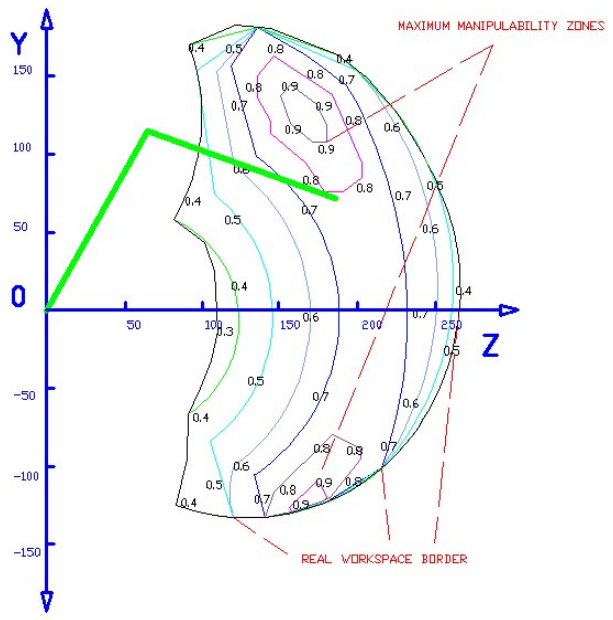

Fig. 3. Manipulability map of PHANToM OMNi for the plane YZ considering movement restrictions. Arms of the manipulator in bold lines. Axes in millimetres. 
Now we must take into account that, due to the mechanical restrictions during its implementation, the device has a limited area of work that includes all those points that it is capable of reaching. We have called Real Workspace (RW) to the extension of useful workspace of the OMNi, obtaining the map of Manipulability showed in Fig. 3. Note that this map extends in the plane YZ and includes the drawing of a RW borderline obtained by measuring manually the OMNi device range of angles $\theta_{2}$ and $\theta_{3}$.

Note that, as it was expected, the OMNi mechanical design includes in the RW the best values of Manipulability.

\section{3D Map of Manipulability}

We can extend the map 2D developed previously by analyzing the behaviour of the device in its whole surrounding space. This RW defined in $3 \mathrm{D}$ is a volume of the space near of the OMNi that contains all points of the space that the End Effector can reach. If we assign to each of points the Manipulability measure calculated by (1), the resultant volume is the 3D map of Manipulability associated to the device.

This map constitutes one important feature of the OMNi that we can consider to be physically joined the device. We can realize this map as a zone of influence of the device in its environment [6].

Figure 4 shows a representation of the Omni device together with its 3D map of Manipulability. Note that it has been limited to the OMNi Real Workspace.

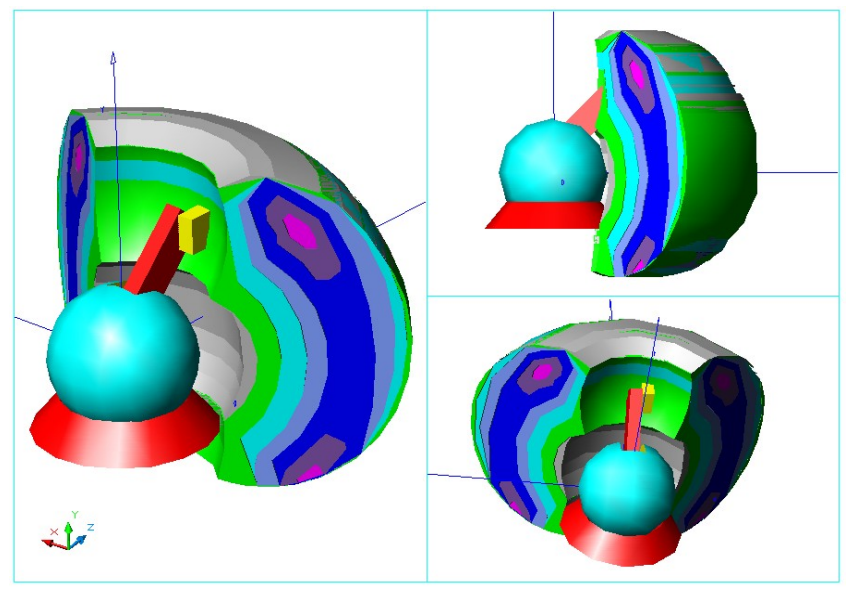

Fig. 4. 3D Map of Manipulability

\section{Measurement of Suitability}

There are several scientific works aimed to develop methods of optimizing the use of manipulators [7]. Some of these works have used the measure of Manipulability as a criterion of optimization [8], [9]. The design of a virtual reality system requires positioning the Application Workspace (AW) inside the 3D map of Manipulability. This 
intersection affect zones with different values of $\mu$ (different colored volumes $v_{i}$ in figure 4). The measure we need must provide the quality of the mechanical positioning of an OMNi respect of the AW.

For a given a positioning of the device in a simulation platform, and therefore an AW divided in different sub-volumes of Manipulability, we define the "Average Volumetric Manipulability" $\mu_{\mathrm{v}}[5]$ as:

$$
\mu_{v}=\frac{\left(\mu_{1} \cdot v_{1}+\mu_{2} \cdot v_{2}+\mu_{3} \cdot v_{3}+\ldots \ldots \ldots \mu_{n} \cdot v_{n}\right)}{V_{T}}
$$

Where:

$\mathrm{V}_{\mathrm{T}}$ is the total volume of the intersection:

$$
\mathrm{V}_{\mathrm{T}}=\sum_{\mathrm{i}}^{\mathrm{n}} \mathrm{v}_{\mathrm{i}}=\mathrm{v}_{1}+\mathrm{v}_{2}+\ldots+\mathrm{v}_{\mathrm{n}}
$$

This measure assigns each sub-volume the weight of its correspondent Manipulability calculating then the average value over the total volume. We can use $\mu_{v}$ for qualifying the configuration of a device (with its RW) respect to a determinate application (with its AW). From our experience using this measure, we can affirm that, if we design a mechanical platform that produces an Average Volumetric Manipulability of a value rounding 0.8 or even 0.9 , we can consider the fitting of the AW inside of RW as excellent.

The practical use of this concept has the difficulty of finding the best mechanical device positioning in a very large space of search.

Our first approach is to use a set of empirical rules in order to help in the searching of a good configuration. Unfortunately using this procedure does not assure we have obtained the best solution.

To solve partially this problem, we have developed an automatic searching process based on Simulated Annealing [10]. Every step of the simulated annealing algorithm the system searches randomly a solution near the current one, according to a probability that depends on the current temperature (similar to the metallurgy concept of Annealing). The algorithm allows calculating an optimal positioning of AW that produces a maximum value of the cost function $\mu_{\mathrm{v}}$.

Studying the possibilities of improving the previous results we have seen that our application has zones of AW which are more used than others. These are, for instance in a surgical operation simulation, the specific areas where the surgical intervention is effectuated. It is desirable that the haptic device provides its best performance in these zones. We propose, for the cases in which the use of the workspace is not homogeneous, to perform an additional analysis taking in account this heterogeneity. It involves the study of the End Effector movement across the AW during the simulation process.

Considering a discretized AW using a tri-dimensional grid of cells $(i, j, k)$, we define a new measure called "Useful Manipulability" for each AW configuration respect to RW using the formula:

$$
\hat{\mu}_{\mathrm{v}}=\sum_{\mathrm{ijk}} \mu_{\mathrm{vijk}} \cdot \mathrm{f}_{\mathrm{ijk}}
$$


Where:

$\mu_{\mathrm{v} i \mathrm{jk}}$ is the Volumetric Average Manipulability of a cell.

$\mathrm{f}_{\mathrm{ijk}}$ is the frequency of visits sampled during a simulation session in each cell.

Note that if the size of cells $(\mathrm{i}, \mathrm{j}, \mathrm{k})$ is small enough we can consider that Manipulability is constant and we can use the more simple formula:

$$
\hat{\mu}_{\mathrm{v}}=\sum_{i j k} \mu_{i j k} \cdot f_{i j k}
$$

Where:

$\mu_{\mathrm{ijk}}$ is the Manipulability of a cell.

With this criterion the best positioning of the OMNi in terms of frequency of use will produce maximum value of $\hat{\mu}_{\mathrm{v}}$.

\section{Results}

We have used the above defined measures to calculate the grade of suitability of a manipulator in a real application. The application consists of the positioning of an OMNi device used as a component of the mechanical platform of a Minimal Invasive Surgery Trainer [1]. We have used a set of simple geometrical shapes to model the internal cavity of a human shoulder (Figure 5). In a previous work we have shown the use of this kind of geometrical shapes to make easier the task of fitting the manipulator RW with the AW [9].

Figure 5 shows the AW corresponding with a virtual model of this AW. The upper part represents the subacromial cavity and the cylindrical sized one situated below represents the glenohumeral cavity. The figure shows two different volumes of intersection between the AW and the 3D map of Manipulability.
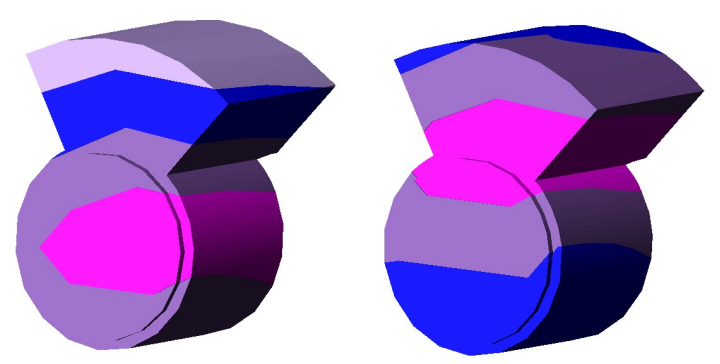

Fig. 5. Two possible configurations of AW respect to OMNi location

Option 1 (Table 1). In this option we have situated the AW in such position that optimizes the operation into the glenohumeral zone. This positioning provides an Average Volumetric Manipulability $\mu_{v}=0.8073$ (fig. 5-left).

After the mentioned process of discretization, considering the frequency of use of the different volumes we have obtained a Useful Manipulability of $\hat{\mu}_{v}=0.8026$. 
Table 1. Option 1. Optimizing glenohumeral zone.

\begin{tabular}{|c|c|c|c|}
\hline $\mathrm{V}_{\mathrm{i}}\left(\mathrm{mm}^{3}\right)$ & Volume Figure & $\mu$ & $\mu_{\mathrm{i}} \cdot \mathrm{v}_{\mathrm{i}}$ \\
\hline $\mathrm{V} 1=11177$ & & $\mu=0.9-1$ & 10786 \\
\hline $\mathrm{V} 2=16269$ & & $\mu=0.8-0.9$ & 14073 \\
\hline $\mathrm{V} 3=22612$ & & $\mu=0.7-0.8$ & 17298 \\
\hline $\mathrm{V} 4=12101$ & & $\mu=0.6-0.7$ & 8047 \\
\hline $\mathrm{V}_{\mathrm{T}=62159}$ & & $\mu_{\mathrm{v}}=\frac{\sum_{\mathrm{i}} \mu_{\mathrm{i}} \cdot \mathrm{v}_{\mathrm{i}}}{2}$ & \multirow{2}{*}{0.8073} \\
\hline
\end{tabular}

Table 2. Option 2. CG at maximum Manipulability zone.

\begin{tabular}{|c|c|c|c|}
\hline $\mathrm{V}_{\mathrm{i}}\left(\mathrm{mm}^{3}\right)$ & Volume Figure & $\mu$ & $\mu_{\mathrm{i}} \cdot \mathrm{v}_{\mathrm{i}}$ \\
\hline $\mathrm{V} 1=16238$ & & $\mu=0.9-1$ & 15426 \\
\hline $\mathrm{V} 2=29282$ & & $\mu=0.8-0.9$ & 24890 \\
\hline$V 3=16639$ & & $\mu=0.7-0.8$ & 12562 \\
\hline$V_{T}=62159$ & & $\mu_{\mathrm{v}}=\frac{\sum_{\mathrm{i}} \mu_{\mathrm{i}} \cdot \mathrm{v}_{\mathrm{i}}}{\mathrm{v}_{\mathrm{T}}}$ & 0,8507 \\
\hline
\end{tabular}


Option 2 (Table 2). This configuration consists of positioning the maximum Manipulability zone in the centre of gravity of the whole AW. This option tries to distribute the available Manipulability between the two operation workspaces. This option provides an Average Volumetric Manipulability: $\mu_{v}=0.8506$ (fig. 5-right).

Considering the frequency of use of the different volumes we have obtained a Useful Manipulability of $\hat{\mu}_{\mathrm{v}}=0.8022$.

Note that the option 2 is the best election in terms of Average Volumetric Manipulability, while the result is similar taking in account the frequency of use. We can say that the improvement of $\mu_{\mathrm{v}}$ in the positioning represented by option 2 has been obtained for zones rarely visited in the course of a normal simulation.

An improvement of this positioning was obtained using an automatic searching process based on Simulated Annealing [11]. With this procedure we have obtained the maximum value of $\hat{\mu}_{\mathrm{v}}=0.8685$. This value corresponds with positioning the centre of gravity of AW at the position XYZ $(-9,130,100) \mathrm{mm}$ from the origin at the centre of the OMNi.

\section{Conclusions}

In this work we analyze the problem of optimize the positioning of a manipulator inside of the application workspace of a virtual reality system.

We have introduced two measures of the quality of this positioning: The concept of Average Volumetric Manipulability is useful to evaluate the performance that we can expect from a specific mechanical positioning of a haptic device in the virtual reality system platform. As a complement of this measure, the Useful Manipulability measures the grade in which the available Manipulability is located in the spatial zones were the manipulator is used more frequently.

The advantage of using these two measures has been demonstrated practically during the design and development of a Minimal Invasive Surgery Trainer.

Acknowledgements. The authors are grateful to the Modeling and Virtual Reality Group (GMRV) of the Rey Juan Carlos University.

This work has been partially funded by the Spanish Ministry of Education and Science (grant TIC2003-08933-C02-01), Government of the Community of Madrid (grant GR/SAL/0940/2004 and grant S-0505/DPI/0235).

\section{References}

1. Bayona, S., Garcia, M., Mendoza, C., Fernandez, J.M.: Shoulder Arthroscopy Training System with Force Feedback, In: International Conference on Medical Information Visualisation-BioMedical Visualisation (MedVis'06)2006, pp. 71-76 (2006)

2. Murray, R.M., Li, Z., Sastry, S.S.: A mathematical introduction to robotic manipulation. CRC Press, Inc, Boca Raton, FL (1994)

3. Yoshikawa, T.: Manipulability and redundancy control of robotic mechanisms, Robotics and Automation. In: Proceedings of IEEE International Conference on (Mar 1985), vol. 2, pp. 1004-1009 (1985) 
4. Cavusoglu, M. C., Feygin, D., Tendick, F.: A Critical Study of the Mechanical and Electrical Properties of the PHANToM Haptic Interface and Improvements for High Performance Control. Teleoperators and Virtual Environments, 11(6), 555-568 (2002)

5. Yoshikawa, T.: Foundations of Robotics: Analysis and Control. MIT Press, Cambridge, MA (1990)

6. Sobh, T.M., Toundykov, D.Y.: Optimizing the tasks at hand [robotic manipulators]. Robotics \& Automation Magazine, IEEE 11(2), 78-85 (2004)

7. Alqasemi, R.M., McCaffrey, E.J., Edwards, K.D., Dubey, R.V.: Analysis, evaluation and development of wheelchair-mounted robotic arms. Rehabilitation Robotics 2005, ICORR 2005. In: 9th International Conference on 28 June-1 July 2005. pp:469-472 (2005)

8. Guilamo, L., Kuffner, J., Nishiwaki, K., Kagami, S.: Manipulability optimization for trajectory generation. Robotics and Automation 2006, ICRA 2006. In: Proceedings 2006 IEEE International Conference on May 15-19, 2006. pp: 2017-2022 (2006)

9. San Martin, J., Trivino, G.: Mechanical performance of a manipulator in virtual reality systems. In: 2nd International Conference on Computer Graphics Theory and Applications. GRAPP 07 March 2007 (Accepted, in press)

10. Kirkpatrick, S., Gelatt, Jr., C.D., Vecchi, M.P.: Optimization by Simulated Annealing, Science (220), 671-680 (13 May 1983)

11. Aragon, C.R., Johnson, D.S., McGeoch, L.A., Shevon, C.: Optimization by Simulated Annealing: An Experimental Evaluation; Part II, Graph Coloring and Number Partitioning. Operations Research 39(3), 378-406 (1991) 